Document downloaded from:

http://hdl.handle.net/10251/99655

This paper must be cited as:

Balador, A.; Tavares De Araujo Cesariny Calafate, CM.; Juan-Carlos Cano; Manzoni ., P. (2017). A density-based contention window control scheme for unicast communications in vehicular ad hoc networks. International Journal of Ad Hoc and Ubiquitous Computing. 24(12):65-75. doi:10.1504/IJAHUC.2017.080913

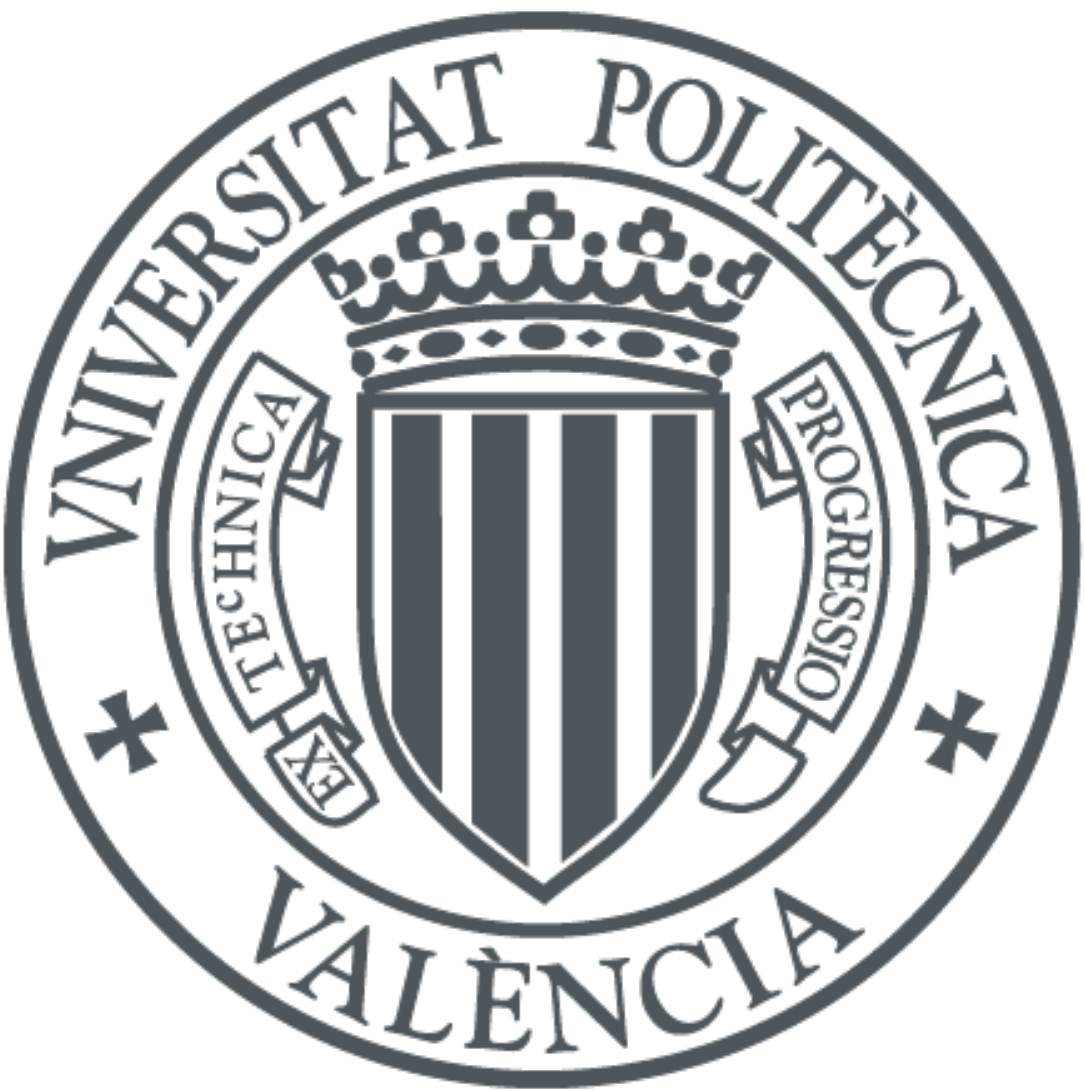

The final publication is available at

https://doi.org/10.1504/IJAHUC.2017.080913

Copyright Inderscience Enterprises Ltd.

Additional Information 


\title{
A Density-Based Contention Window Control Scheme for Unicast Communications in Vehicular Ad Hoc Networks
}

\author{
Ali Balador \\ Universitat Politècnica de València \\ Camino de Vera, s/n, 46022 Valencia, Spain \\ E-mail: alba6@upv.es \\ Corresponding author
}

\section{Carlos Calafate}

Universitat Politècnica de València

Camino de Vera, s/n, 46022 Valencia, Spain

E-mail: calafate@disca.upv.es

\section{Juan-Carlos Cano}

Universitat Politècnica de València

Camino de Vera, s/n, 46022 Valencia, Spain

E-mail: jucano@disca.upv.es

\section{Pietro Manzoni}

Universitat Politècnica de València

Camino de Vera, s/n, 46022 Valencia, Spain

E-mail: pmanzoni@disca.upv.es

\begin{abstract}
Vehicular Ad Hoc Networks (VANETs) have attractive potential in order to reduce traffic jams and avoid transportation disasters. They are also able to provide infotainment services like web browsing, e-mail, or using social networks on the road. Achieving a well-designed Medium Access Control (MAC) protocol is a challenging issue to improve communications efficiency due to the dynamic nature of VANETs. The CSMA-based MAC protocol adopted by the IEEE $802.11 \mathrm{p}$ standard was selected as the best choice for the current generation of vehicular ad hoc networks considering its availability, maturity, and cost. Despite these benefits, the common problem in all IEEE 802.11 based protocols is scalability, exhibiting performance degradation in highly variable network scenarios. Experimental results for the IEEE 802.11-based MAC protocol show the importance of contention window adjustment on communications performance; However the vehicular communications community has not yet addressed this issue in unicast communication environments.

This paper proposes DBM-ACW, a novel contention window control scheme for VANET environments based on estimating the network density, which is then used to dynamically adapt the $\mathrm{CW}$ size. Analysis and simulation results using $\mathrm{OMNeT}++$ in vehicular scenarios, including highway and urban scenarios, show that DBM-ACW provides better overall performance compared with previous proposals, even in high network density scenarios.
\end{abstract}

Keywords: IEEE 802.11p; Contention Window; Density Estimation; Unicast Communication; Vehicular Ad Hoc Networks

Reference to this paper should be made as follows: Balador, A., Calafate, C., Cano, J-C. and Manzoni, P. (xxxx) 'A Density-Based Contention Window Control Scheme for Unicast Communications in Vehicular Ad Hoc Networks', International Journal of Ad Hoc and Ubiquitous Computing, Vol. x, No. x, pp.xxx-xxx.

Biographical notes: Ali Balador is currently a PhD Student at the GRC research group, Universitat Politcnica de Valncia, under supervision of Dr. Juan Carlos Cano, and Dr. Carlos Calafate. He received his B.Sc. and M.Sc. degrees in Computer Engineering from the Islamic Azad University in 2007 and 2010, respectively. His research interests include wireless networks, network simulation, vehicular ad-hoc networks, and MAC layer protocols. 


\section{Introduction}

Achieving a transportation system without disasters or traffic jams has been a dream of the research community for several decades. With advances in wireless communication technologies, VANETs have emerged as an attractive research area for both academia and industry. Vehicular networks are formed by vehicles equipped with wireless devices, called On-Board Units (OBUs), which allow communicating with other vehicles in infrastructure-less wireless networks (vehicleto-vehicle, V2V) or with roadside units (vehicle-toinfrastructure, V2I). Vehicular environments integrating inter-vehicle communications can be assumed as a special form of Mobile Ad-Hoc Networks (MANETs) in which node's mobility is road-constrained (Hartenstein and Laberteaux, 2008).

The main differences between VANETs and MANETs have to do with rapid topology changes and diversity of network scenarios. Also, it must be taken into account that, in contrast to MANETs, vehicle's density is highly dynamic (Zeadally et al., 2012). For example, in highway scenarios, vehicles have mostly one straightforward direction, but high relative speeds up to $100 \mathrm{~km} / \mathrm{h}$, compared to the cars driving in the opposite direction that can lead to a challenging situation. On the other hand, in urban scenarios, high-density networks appear during rush hours. Also, network disconnection can occur in the late night hours or idle daytime hours. Therefore, designing protocols able to take all of these characteristics into account is still a challenging open issue.

Medium Access Control protocols play an important role since critical communications rely on them. Unfortunately, research results in Booysen et al. (2011) highlight that the topic of MAC support in VANETs has received less attention than in other research fields. Also, most of the relatively few research works are dedicated to V2I communications; therefore, MAC support for V2V communication needs more attention. MAC layer design challenges in VANET environments can be summarized as follows (Kenney, 2010): (a) Achieving an effective channel access coordination in the presence of changing vehicle locations and variable channel characteristics; (b) supporting scalability in the presence of various traffic densities; and (c) supporting a diverse set of application requirements.

According to the definition in Clifford Neuman (1994), a system is said to be scalable if it can handle the addition of users and resources without suffering a noticeable loss of performance or increase in administrative complexity. Due to the high dynamics in vehicle's density in vehicular environments, the MAC protocol must be scalable in order to fulfil end-to-end delay and packet delivery ratio requirements.

Also, as mentioned, good VANET protocol designs should account for different kinds of applications. Applications for VANETs can be categorized into three groups: safety, convenience (traffic efficiency), and commercial applications (infotainment) (Bai et al., 2006); each of these classes of applications has its own QoS requirements. Safety applications increase each vehicle's awareness about its neighbourhood by broadcasting small messages to a close neighbourhood, so that these messages are geographically limited. The target goals of traffic efficiency applications are basically to reduce the time each vehicle spends on the road in order to reduce fuel consumption and air pollution. Finally, the third group of applications consist of infotainment applications. Although they have no effect on road traffic, they provide convenience and comfort services to drivers and/or passengers. In the latter, delay is not as critical in comparison to safety applications, but the transferred data volume is much larger.

IEEE 802.11p (IEEE 802.11p Std, 2010) is an amendment to the IEEE 802.11 for vehicular networks, also called Wireless Access in Vehicular Environments (WAVE). The IEEE $802.11 \mathrm{p}$ proposes physical layer changes, while the MAC layer has remained mostly the same as in IEEE 802.11.

Different solutions have been proposed by the research community in order to improve the performance of IEEE $802.11 \mathrm{p}$ concerning safety applications, e.g (Asadallahi and Refai, 2012; Omar et al., 2012; Stanica et al., 2011; Wang et al., 2012), which basically deal with the use of broadcast communication. However, the results in Bai et al. (2006) confirm that some traffic efficiency applications, and also the majority of infotainment applications, rely on unicast communications. This classification clarifies that, although safety applications have attracted special interest and require more scrutiny, other types of applications need to be attended as well. Also, as shown by authors such as Cottingham et al. (2007); Jansons and Barancevs (2012); Wellens et al. (2007), vehicular ad hoc networks introduce complex dynamics that have not emerged in other types of MANETs. Due to this lack of solutions for non-safety applications, this paper targets unicast communications, which is required by non-safety applications in vehicular scenarios.

Since the IEEE 802.11 MAC protocol was basically designed for static wireless networks, it achieves poor performance in VANET environments. A wellknown problem in IEEE 802.11-based MAC protocol is scalability, which becomes more challenging in VANETs in the presence of high and variable network densities, as a result of higher mobility. Several works have been proposed and carried out for MANET environments (Bharghavan et al., 1994; Bianchi and Tinnirello, 2003; Bononi et al., 2004; Cali et al., 2000; Heusse et al., 2005; Qiang et al., 2003; Song et al., 2003; Wu et al., 2002) to either solve or reduce this problem, but none has adequately addressed this issue in vehicular environments.

In this paper we propose a new Contention Window (CW) control scheme, called DBM-ACW (Density Based Method for Adjusting the CW size). DBM-ACW estimates the channel density by observing and keeping 
a history of channel transmission states, and then adapts the CW size based on the density estimations. We demonstrate that DBM-ACW not only outperforms IEEE $802.11 \mathrm{p}$, but also previously proposed schemes under different vehicular scenarios.

The rest of this paper is organized as follows. In Section II, we briefly explain the basic principles of IEEE 802.11-based MAC protocols, while Section III includes some studies related to this paper and the motivation behind our approach. In Section IV we present our proposed approach in detail, clearly identifying the contributions of this paper compared with previous works. Section V describes the simulation scenarios, the measurements, and the selected metrics for performance evaluation. Simulation results are provided in section VI, including results in both highway and urban scenarios. Finally, Section VII concludes the paper.

\section{IEEE 802.11 and 802.11p Overview}

Although initially proposed for Wireless LAN environments, the IEEE 802.11 standard (ANSI/IEEE Std 802.11, 2003) has expanded in order to fulfill the communication requirements of different environments. IEEE 802.11-based MAC protocols rely on the carrier sense multiple access with collision avoidance (CSMA/CA) mechanism, so that each node listens to the channel before transmission in order to prevent collisions. Since they cannot guarantee a collisionfree MAC, two main mechanisms are proposed to handle medium access collisions among nodes while also avoiding the hidden node problem. First, the Requestto-Send (RTS)/Clear-to-Send (CTS) mechanism is proposed in order to reserve the medium by sending small packets before transmitting large data packets, basically to avoid the hidden node problem. However, due to the high overhead involved when using this mechanism, it is inactive by default. Second, each node must select a random time (called backoff) before sending each packet to avoid packet collisions. Also, IEEE 802.11 proposes three different Inter Frame Space (IFS) time intervals, such as SIFS, DIFS, and PIFS, in order to prioritize access to the wireless channel.

When a new data packet is waiting in the buffer for transmission, the node is allowed to transmit the packet if it finds the channel free after waiting for a time equal to DIFS. Otherwise, if the channel is found busy after this period, it chooses a backoff value and must wait before attempting to transmit again. The next time the channel is found idle, the node must wait for a time equal to DIFS, plus a backoff time before transmission. The Binary Exponential Backoff (BEB) algorithm uniformly selects the backoff time from the interval $(0, \mathrm{CW})$. The IEEE 802.11 standard initializes the contention window to the predefined value, $C W_{\text {min }}$, and doubles it upon transmission failures up to the predefined value, $C W_{\max }$. Also, The $\mathrm{CW}$ is reset to the $C W_{\min }$ by a successful transmission. The node senses the channel and, if it finds the channel idle, decreases the backoff timer by one; otherwise it pauses the timer. The backoff timer is resumed when the channel again remains idle for a period longer than DIFS.

IEEE 802.11 uses positive acknowledgements (ACK) to confirm the correctness of the current transmission to the transmitter. Such mechanism is mandatory since wireless interfaces cannot transmit and listen to the channel simultaneously. Notice that its own signal is too strong, masking any other incoming signals, and so collision detection becomes very difficult. Second, collision detection at the transmitter side does not allow to infer about collisions at the receiver side. Therefore, if the transmitter cannot receive a correct ACK packet, it considers that a collision has occurred, and the current data packet must be retransmitted (up to a predefined number of times). After receiving a correct data packet, the receiver waits for a SIFS time and sends an ACK to the transmitter.

As previously mentioned, IEEE 802.11 introduces specifications for wireless networks, but it fails to maximize performance in vehicular environments. The main standards and specifications for vehicular environments are short-range communications (DSRC) (Kenney, 2011), IEEE 802.11p (IEEE 802.11p Std, 2010), and IEEE 1609 (Grafling et al., 2010). DSRC includes specifications and standards for communications between vehicles in close neighborhood. Both US and Europe have allocated spectrum in the $5.9 \mathrm{GHZ}$ range for DSRC. IEEE developed IEEE $802.11 \mathrm{p}$, which is an amendment to the original IEEE 802.11 standard for DSRC to better support vehicular communications. IEEE $802.11 \mathrm{p}$ defines specifications for both the link and the physical layers, while IEEE 1609.4 specifies higher layers that will operate on top of IEEE 802.11p. IEEE 802.11p is an IEEE 802.11-based MAC protocol offering a priority scheme in a similar way to IEEE 802.11e EDCA (IEEE Std 802.11e, 2005), while IEEE 1609.4 manages channel coordination and supports MAC service data unit delivery. IEEE $802.11 p$ proposed a multi-channel operation so that the physical layer consists of seven 10MHZ channels, where one of them is the control channel $(\mathrm{CCH})$, used for safety communications, and the remaining are called Service Channels (SCH), and they are used for non-safety applications, as shown in Figure 1.

An Orthogonal Frequency Division Multiplexing (OFDM) modulation scheme is used to multiplex data, similarly to the IEEE 802.11a standard. However, the bandwidth which is used in each channel by IEEE 802.11p is half of the bandwidth used in the IEEE 802.11a. The MAC layer follows the same approach as used by the IEEE 802.11e EDCA. The EDCA mechanism defines four different access categories (AC) for each channel compared to just one in IEEE 802.11. Different access categories provide different access priorities, and based on that they are assigned different contention parameters. For example, AC3, which has the highest priority when accessing medium, has the 
Figure 1: DSRC Frequency Plan.
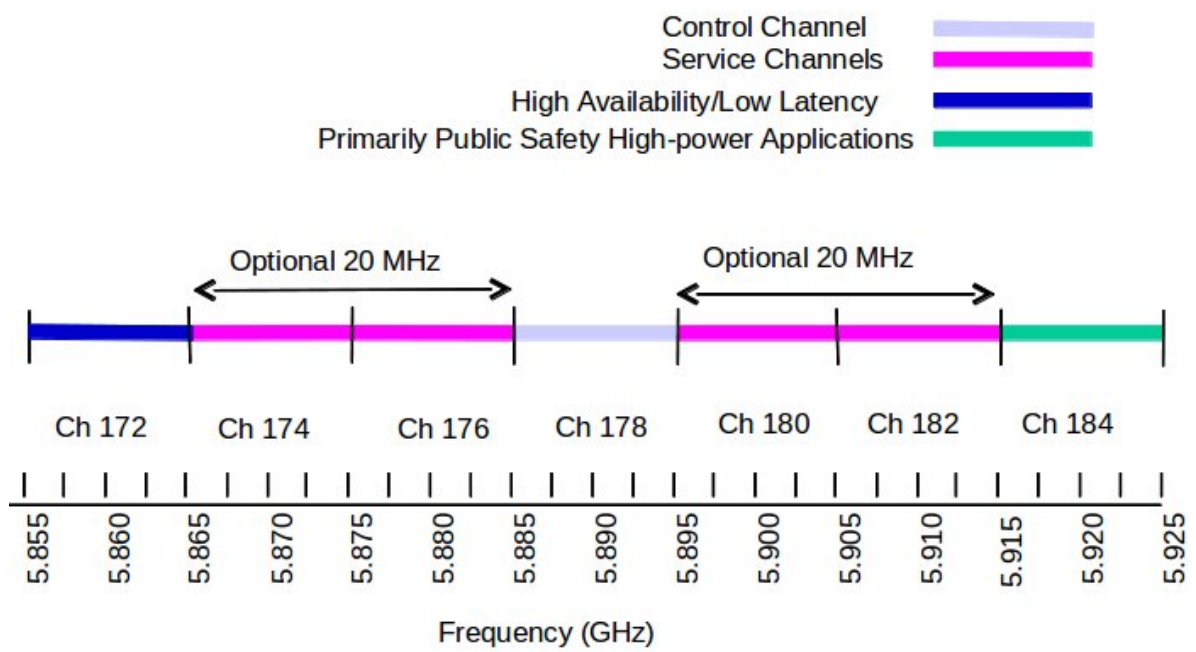

Table 1 Different Application Categories in IEEE 802.11p.

\begin{tabular}{llll}
\hline AC & CWmin & CWmax & AIFSN \\
\hline \hline Video Traffic (AC3) & 3 & 7 & 2 \\
Voice Traffic (AC2) & 3 & 7 & 3 \\
Best Effort (AC1) & 7 & 255 & 6 \\
Background (AC0) & 15 & 1023 & 9 \\
\hline
\end{tabular}

lowest Arbitrary Inter Frame space (AIFS) and CW values, whereas $\mathrm{AC} 0$, which has the lowest priority, has the highest values. Table 1 shows the default parameter settings used in IEEE 802.11 p for different traffic types. Then, there are six service channels and one control channel, and each of them has four different access categories. So, there are two contention procedures including: internal contentions between different access categories in each channel, and the contention between channels to access the medium.

Despite IEEE $802.11 \mathrm{p}$ provides priority differentiation through IEEE 802.11e, it still experiences the common problem of all IEEE 802.11-based MAC protocols, which is the lack of ability to dynamically adapt the CW size. The current IEEE 802.11p standard, which applies to communication in wireless vehicular environments, does not propose an explicit mechanism in order to dynamically adapt the CW size based on the network density. Research results focusing on IEEE 802.11p performance show that this issue is also more challenging in vehicular environments because of higher mobility and higher node density compared to the previously existing wireless networks.

\section{Related Work and Motivation}

To solve the problem of IEEE 802.11-based MAC protocols, some schemes (Bianchi and Tinnirello, 2003; Bononi et al., 2004; Cali et al., 2000; Heusse et al., 2005) have been proposed in which the network density is estimated, and then the $\mathrm{CW}$ size is chosen based on that network density estimation. The AOB mechanism (Bononi et al., 2004) measures the network contention level by using two simple values: the slot utilization, and the average size of transmitted frames, based on the information provided by the carrier sensing mechanism. In Cali et al. (2000), a three level estimator is used for computing the optimal CW size, and in Bianchi and Tinnirello (2003), a method was proposed to estimate the number of active nodes by means of a Kalman filter to optimize the CW size. Also, in Heusse et al. (2005), a novel access method was proposed called Idle Sense that allows adjusting the CW size based on the network load. The $\mathrm{CW}$ is derived after measuring the number of idle slots between two transmission attempts, and comparing it with the optimal number, which is equal to 5.68 for IEEE $802.11 \mathrm{~b}$. Considering the high overhead of these methods to estimate the network density, these approaches are not highly acceptable in ad hoc networks where power and memory resources are restricted.

In order to avoid the high overhead of density estimation, other schemes (Bharghavan et al., 1994; Qiang et al., 2003; Song et al., 2003; Wu et al., 2002) introduce static mechanisms to optimize the $\mathrm{CW}$ size selection instead of harshly changing the $\mathrm{CW}$ size by resetting it to the minimum value (or doubling it) upon a successful or unsuccessful transmission, respectively. For example, the MILD scheme (Bharghavan et al., 1994) increases the CW size by multiplying it by 1.5 and decreasing the CW size by one unit. In Wu et al. (2002), when the retry counter reaches the limit, the $\mathrm{CW}$ is not set to the minimum CW. After a successful transmission, the $\mathrm{CW}$ is set to the value $\max \left[C W / 2, C W_{\min }+1\right]$ and, upon a transmission failure, $\mathrm{CW}$ is set to the value $\min \left[2 C W, C W_{\max }+1\right]$. EIED, which is proposed by Song et al. (2003), modifies the performance of BEB and MILD under low traffic conditions. The EIED scheme is based on an exponential distribution for both increasing and decreasing the CW size. In Qiang et al. 
(2003), The new scheme is proposed which, following a successful transmission, reduces the $\mathrm{CW}$ to a value near the old one based on some factor that is evaluated during simulation. While these schemes work better than the $\mathrm{BEB}$ algorithm for high density networks, the basic problem remains since the static behaviour persists, and so it cannot adapt to different network densities. In Aad et al. (2004), the authors evaluate different methods for slowly decreasing the $\mathrm{CW}$ size from the data rate, delay, response time, fairness, and power saving efficiency point of view. They showed that avoiding to reset the $\mathrm{CW}$ size to $C W_{\min }$ can significantly improve the overall performance.

To fill the gap between the two aforementioned groups of methods, a low-overhead and dynamic solution called HBCWC is proposed by Balador et al. (2010). This algorithm does not need complex computations to estimate the network density. Instead, HBCWC obtains the network density information by observing the channel status, and then it dynamically adapts the $\mathrm{CW}$ size based on the channel condition history. This approach exhibits a significant improvement in the presence of high network densities, while not requiring significant changes to the original IEEE 802.11 MAC. Although HBCWC achieves significant improvements, it still sets the CW size to $C W_{\min }$ upon a successful transmission, which causes some performance degradation.

In summary, it becomes clear that modifying IEEE 802.11p MAC protocols for unicast communications in vehicular environments is a requirement when attempting to offer full support for all types of VANET applications. To the best of our knowledge, very few studies address this subject. For example, a fuzzy logic based enhancement to the $802.11 \mathrm{p}$ mechanism is proposed by Chrysostomou et al. (2011) which adapts the $\mathrm{CW}$ size based on a non-linear control law by relying on channel observation. Therefore, the purpose of this paper is to propose a new solution for the IEEE 802.11p MAC protocol by taking into account the advantages of previous approaches, in order to tune the $\mathrm{CW}$ size based on the network density, and considering the computational overhead in different vehicular scenarios.

\section{DBM-ACW: Our Density Based Method for Adjusting the CW Size Scheme}

As mentioned above, in IEEE 802.11-based MAC protocols, a significant reduction of the network performance, especially in dense traffic networks, stems from the lack of ability to select an optimal CW size. To solve this problem, we propose a new method, DBM$\mathrm{ACW}$, to select the $\mathrm{CW}$ size based on the network traffic density. In this method, the channel conditions are observed based on the packet transmission status, and the result is stored into a Channel State (CS) vector. This vector must be updated after each transmission attempt. A significant part of the protocol relies on how

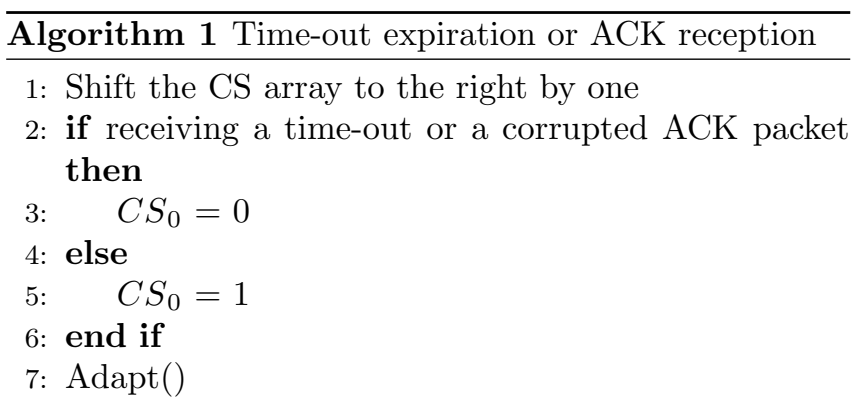

the channel condition is captured by the CS vector, and how this vector is then used to update the CW size in order to improve throughput, which is the key contribution of this paper. These two issues will be further explained in the following sections.

\subsection{Initialization}

The CS vector, that is introduced by our protocol and used for keeping track of channel conditions, is initially set to one in order to assume a collision free environment. The proposed approach relies on a set of parameters to optimally adapt the CW size to network density, which are further used in Algorithm 2. These parameters must be set in this step. A more detailed discussion on how these parameters were obtained is presented in section 4.3 .

\subsection{The Channel State Vector}

In 802.11-based MAC protocols, and after each data frame transmission, each node sets its timer and waits for an acknowledgement. In DBM-ACW, upon each timer expiration, or upon receiving a packet, Algorithm 1 is called. This algorithm basically behaves as follows: if the transmitter receives an ACK frame from the receiver, a value of 1 is inserted into the channel state (CS) vector (Line 5); otherwise, if a collided/faulty frame is received, or if the transmitter waiting timer expires before receiving the acknowledgement, a value of 0 is inserted into the CS vector (Line 3). Note that we assumed that one service channel is assigned for our application, therefore, the broadcast traffic in the control channel cannot affect the performance of our protocol.

The CS vector is updated by shifting before setting the $C S_{0}$ value (Line 1 ). After setting the CS vector, function Adapt (Algorithm 2) is called in order to adapt the CW. Based on extensive simulation results, we chose a three-element array in order to achieve a trade-off between overhead and performance. If a smaller array is chosen, it will fail to assess the real network conditions, while larger array values do not lead to significant performance improvements.

\subsection{Changing the Contention Window Size}

Upon each timer expiration or packet reception, Algorithm 2 is called in order to update the value of the 


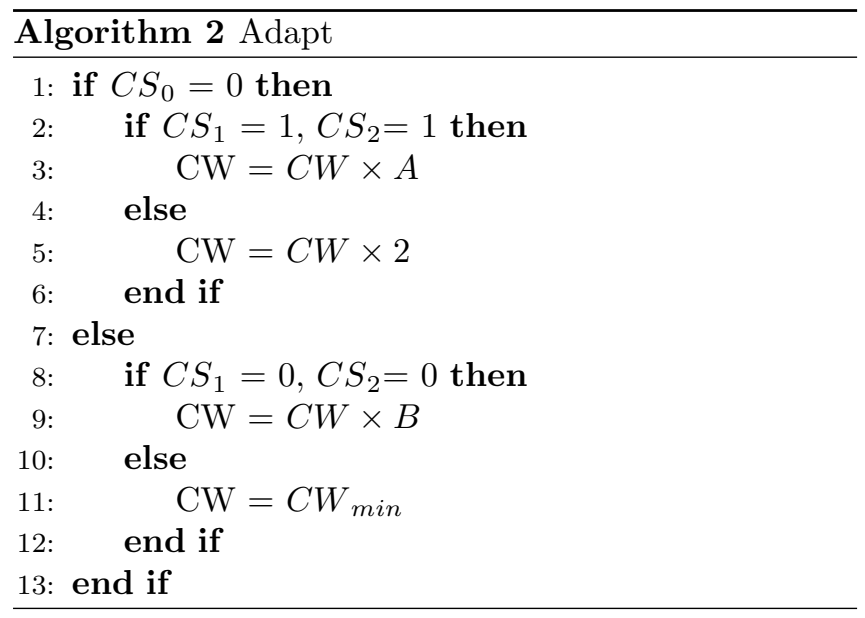

CW. Upon each packet loss, timer expiration or collision, the CW size is increased. DBM-ACW multiplies the CW value by 2 in order to obtain the highest PDR, except for the case in which the CS array contains two consecutive ones before the new state; that particular situation means that we observed two successful transmissions before detecting an unsuccessful transmission. In that case the CW is multiplied by parameter A (Line 3).

In DBM-ACW, the CW size is set to the minimum $\mathrm{CW}, C W_{\min }$, upon each acknowledgement reception, except for the case in which the CS array contains two consecutive zeros before the new state, which means that two unsuccessful transmissions are observed before detecting a successful transmission. In that case the $\mathrm{CW}$ is multiplied by parameter B (Line 9).

Algorithm 2 details how the CW size is chosen in our DBM-ACW scheme. Depending on the channel congestion severity, the current $\mathrm{CW}$ size is multiplied by a value in the range from 0.2 to 2 , or set to $C W_{\min }$. The upper bound is selected as in IEEE $802.11 \mathrm{p}$, so that the CW size is multiplied by 2 when the channel is detected as busy or a collision has occurred. When the channel is very congested, the current $\mathrm{CW}$ size is multiplied by a value in the range from 1.1 to 2 in order to decrease the probability of selecting the same backoff number. Otherwise, when the channel density is low, the current $\mathrm{CW}$ size is multiplied by a value in the range from 0.2 to 1.1 , or set to $C W_{\min }$ in order to avoid waiting for a long time when channel occupation is low. The parameter values can be controlled based on the network traffic density during the simulation but, in order to decrease the protocol overhead, we decided to fix these values before the simulation. Therefore, we repeated the simulation using different combinations of values to obtain the best performance. Based on extensive simulations, the optimal value for parameters $\mathrm{A}$ and $\mathrm{B}$ was found to be equal to 1.7 and 0.8 , respectively.

\section{Simulation Environments}

In this section, we study the performance of DBM-ACW in comparison with the IEEE 802.11p and HBCWC
(Balador et al., 2010) in vehicular environments by using OMNeT ++ (version 4.2.2). OMNeT ++ is a $\mathrm{C}++$ based, open source, and discrete event network simulator with strong GUI support. Since OMNeT++ is a simulation framework, it does not provide models for wireless network simulation. Therefore, we chose this simulator coupled with the INETMANET framework and SUMO (Behrisch et al., 2011) in order to provide a realistic vehicular scenario. The INETMANET framework provides detailed models for simulating wireless networks in $\mathrm{OMNeT}++$ such as wireless channels, connectivity, mobility, and MAC layer protocols. Also, SUMO is used to generate real vehicular traffic in road networks. A SUMO network file describes the roads and intersections where the simulated vehicles run along or across. Vehicles enter from an intersection and then randomly move on streets inside the predefined simulation area. A trip defines the departure time and the destination edge, while a route expands a trip by defining all the edges through which a vehicle will pass. Routes are generated by the shortest path algorithm by taking into account several data sources such as street length, speed limit, lane count, and street type for the shortest path computation. Vehicles in $\mathrm{OMNeT}++$ move based on the trace file which is generated by SUMO. Also, for the car following model, we used the Kraußmodel.

In order to study the efficiency of DBM-ACW, we evaluate its performance in both highway and urban scenarios. The general simulation parameters are as follows: each vehicle generates constant bit rate traffic using UDP datagrams (we chose UDP and not TCP for the same reasons stated in Cottingham et al. (2007)). 512-byte datagrams were transmitted at a rate of 4 packets per second. Considering the routing protocol, we assessed different routing protocols (i.e., AODV, OLSR, DYMO, DSR) and, despite of the different overall performance levels obtained by these protocols, they have the same impact on the different MAC protocols evaluated in this paper. Thus, the results presented in this paper refer to the AODV routing protocol; notice that, since several researchers use this protocol as well, it makes comparison against other proposals easier. To accurately model real world conditions, we used the radio propagation model presented in (Baguena et al., 2012). The transmission range was set to $250 \mathrm{~m}(-85 \mathrm{dBm})$ and the interference range is up to $4 \mathrm{~km}(-110 \mathrm{dBm})$ (Eichler, 2007). Table 2 summarizes the simulation parameters.

The metrics used to evaluate the performance of the MAC solutions under study were similar to those used in previous studies. They are summarized as follows: (a) Packet Delivery Ratio (PDR), which represents the ratio of the total number of packets received by the final destination and the packets originated by the source; (b) average end-to-end delay, which represents the average time required for a packet to travel from source to destination; (c) Standard Deviation of end-to-end delay, which shows how much variation exists from the average end-to-end delay; and (d) average MAC collisions, which 
Figure 2: Valencia real urban scenario.

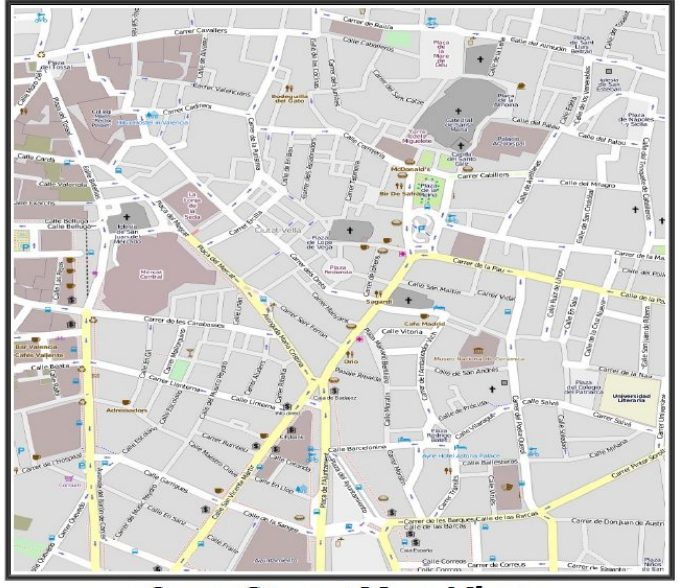

OpenStreetMap View

Table 2 The simulation parameters

\begin{tabular}{ll}
\hline Simulation Parameter & Value \\
\hline \hline Traffic type & CBR \\
CBR packet size & 512 byte \\
CBR data rate & 4 packet/s \\
Transport protocol & UDP \\
Routing protocol & AODV \\
\hline MAC protocol & $802.11 \mathrm{p}$, DBM- \\
& ACW,HBCWC \\
Max. and Min. of CW & 7,1023 \\
Max. number of retransmissions & 7 \\
Max. queue size & 14 \\
RTS/CTS threshold & 2346 byte \\
Slot time & $13 \mu \mathrm{s}$ \\
\hline Simulation time & 300 seconds \\
Number of repetitions & 10 \\
\hline
\end{tabular}

shows the average number of collisions experienced per source.

Each point in the figures that follow represents the average of 10 independent simulation experiments.

\section{Result and Discussion}

In order to evaluate the protocols in vehicular ad hoc networks, two common scenarios that come to mind are highway, and urban scenarios. While the traffic is homogeneous in one-dimensional highway scenarios, urban scenarios show a two-dimensional traffic pattern, which is more complex and challenging.

\subsection{Urban scenarios}

In contrast to highway scenarios, where vehicles always drive in a same direction, and where obstacles are mostly non existent, urban scenarios offer more flexibility in terms of mobility, but also introduce more obstacles like buildings, vehicles, urban furniture, etc (Bohm et

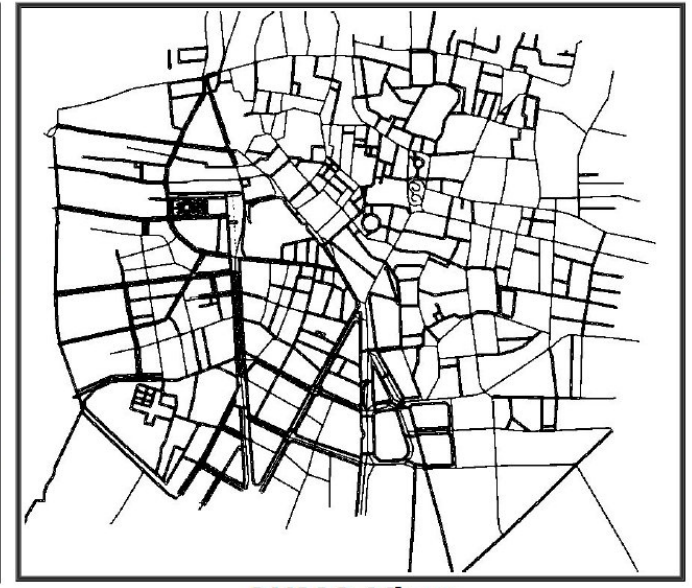

SUMO View

al., 2010; Martinez et al., 2013; Sommer et al., 2011). This issue leads to lower transmission ranges for urban scenarios. As described above, we used SUMO and connected it to $\mathrm{OMNeT}++$ in order to generate realistic urban mobility traces. The urban scenario represents an area of $1,500 \times 1,500 \mathrm{~m}^{2}$ that is extracted from the downtown area of Valencia (Spain) by using digital maps freely available in OpenStreetMap, and including real obstacles. Figure 2 shows two map views: the OpenStreetMap view and the SUMO view.

The vehicle and mobility generation are handled by SUMO. However, when a vehicle reaches its destination in SUMO, it must leave the simulation, and so we cannot ensure a constant number of vehicles throughout the entire simulation time. Therefore, the VACaMobil tool by Baguena et al. (2013) is used to handle this issue, inserting new vehicles in the simulation when other vehicles leave it, thereby maintaining the same number of vehicles throughout the simulation time. In our experiments, the number of nodes varies from 50 to 200.

In this first experiment, we evaluate DBM-ACW in an urban scenario using V2V communications. Since we are interested in high congestion environments, we define a large number of connections, so that each vehicle, immediately after joining the network, starts a new connection and maintains it active until the destination leaves the network. When this occurs, a new destination will be chosen by the transmitter. This experiment represents a stressing situation for a MAC protocol due to the large number of simultaneous connections. Thus, the experimental setup is adequate to assess how DBM$\mathrm{ACW}$ is able to overcome a high number of collisions to obtain a suitable throughput.

Figure 3 shows the PDR for DBM-ACW when varying the number of source nodes. This figure shows that DBM-ACW outperforms both IEEE 802.11p and HBCWC. We can observe that the improvement ratio in high density networks (more than 100 nodes) is 
higher than for low density networks. This stems from the fact that DBM-ACW avoids resetting the $\mathrm{CW}$ to the minimum value, and mostly maintains the average CW size at high values in the presence of frequent collisions. Therefore, it is able to decrease the number of dropped packets but, under low densities, when the collision frequency is low, the degree of efficiency is not comparable to high density situations. Also, HBCWC shows a good performance because it also estimates the network density to choose the optimal CW size. However, the results show that resetting the $\mathrm{CW}$ size upon a successful transmission, like 802.11 p does, has a significant cost in terms of PDR.

The average number of MAC collisions, shown in Figure 4, offers a hint on how to achieve improvements in terms of PDR. As can be observed in this figure, DBMACW shows that the optimal $\mathrm{CW}$ was chosen so that it decreases the probability of picking the same backoff value, and consequently the number of collisions.

One of the key differences between IEEE 802.11p and our approach is that $802.11 \mathrm{p}$ resets the CW size to the minimum value when the retransmission limit is reached, without taking into account that this event is possibly associated to channel collisions; thus, it assigns a minimum CW size for the next packet. Considering this behaviour, one can expect that the new packet will have lower success chances in order to be sent, and will also need more retransmissions on average. Therefore, our approach achieves lower end-to-end delay than the IEEE $802.11 \mathrm{p}$ standard, as shown in Figure 5.

Figure 5 evidences the difference between our approach and HBCWC, which is further clarified in Table 3. In particular, we avoid resetting the CW size for the case in which we observed two consecutive successful transmissions before detecting an unsuccessful transmission. As a result, we are able to achieve

Figure 3: PDR for the urban scenario.

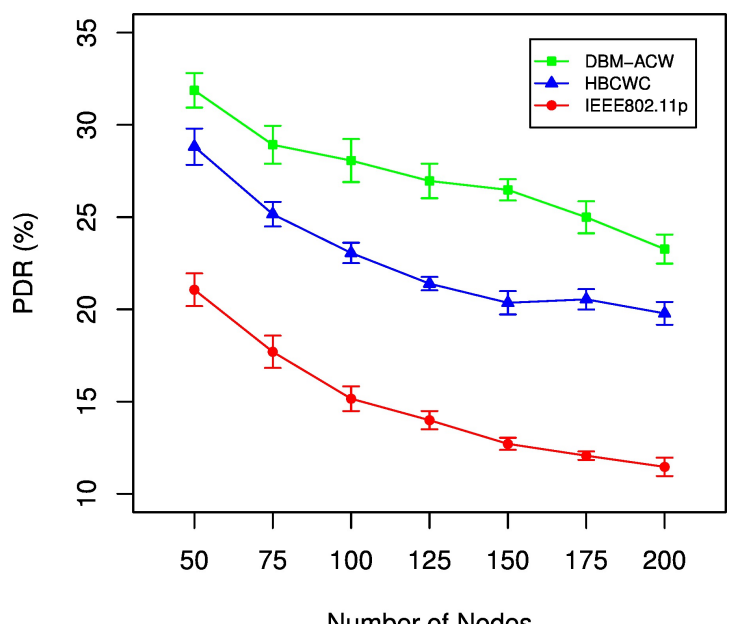

Number of Nodes
Figure 4: Average number of collisions for the urban scenario.

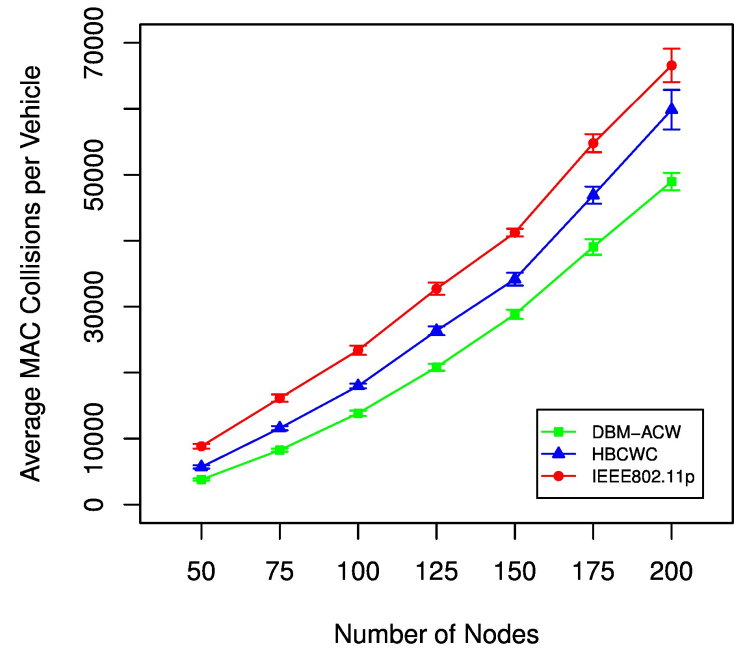

Figure 5: Average end-to-end delay for the urban scenario.

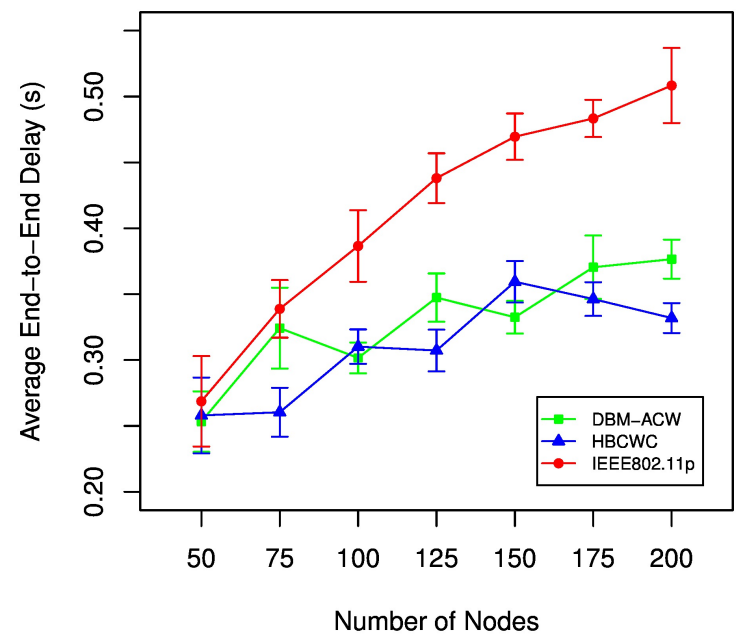

improvements in terms of end-to-end delay, as well as an improved standard deviation for delay when comparing DBM-ACW with HBCWC. In HBCWC, a few packets are sent with very low delay, and this issue decreases the total end-to-end delay, while in DBM-ACW most of the packets have delays close to the average value.

Moreover, as shown in Figure 5, end-to-end delay under high densities does not follow the same trend as 802.11p does, showing a higher bound for delay so that, as the network density increases, the average end-toend delay values remain low, fluctuating at values close to 0.35 seconds. Therefore, we find that DBM-ACW 
Table 3 Standard Deviation of delays for the urban scenario.

\begin{tabular}{llllllll}
\hline & $\mathbf{5 0}$ & $\mathbf{7 5}$ & $\mathbf{1 0 0}$ & $\mathbf{1 2 5}$ & $\mathbf{1 5 0}$ & $\mathbf{1 7 5}$ & $\mathbf{2 0 0}$ \\
\hline \hline DBM-ACW & 0.42 & 0.45 & 0.46 & 0.47 & 0.49 & 0.52 & 0.53 \\
HBCWC & 0.44 & 0.44 & 0.48 & 0.50 & 0.55 & 0.54 & 0.53 \\
\hline
\end{tabular}

improves IEEE $802.11 p$ scalability, which represents an important challenge in vehicular ad hoc networks.

Overall, our approach improves the PDR by $47 \%$, and the end-to-end delay by $16 \%$ when compared with IEEE 802.11 , while also improving the PDR by $16 \%$ in comparison with HBCWC.

\subsection{Highway scenarios}

The highway scenario models a $4 \mathrm{~km}$ highway with 3 lanes, where the lane width is $3 \mathrm{~m}$. As assumed in the previous section, the destination is randomly chosen, so it can be a car ahead or behind of the transmitter. In order to model a realistic scenario, we assumed that vehicles can be selected based on a normal distribution from three different categories that are summarized in Table 4. Sigma models the driver's imperfection which is selected in the range from zero to one. The drivers imperfection shows a lack of perfection in the driver's ability to hold the desired speed. Moreover, vehicles are injected in the highway according to a Poisson process with a mean interval time of 2 seconds, and where the best lane is assigned to each vehicle. In contrast to the urban scenario, the transmission rate is 2 packets per second. Figure 6 depicts the highway scenario. In each graph, the probability of sending a message defines the transmission probability for each vehicle throughout the simulation.

Figure 6: The highway scenario.

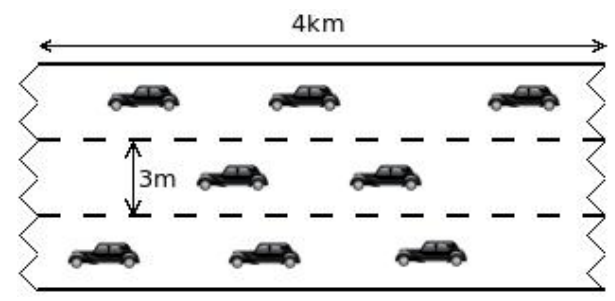

Table 4 The Vehicle Types

\begin{tabular}{llllll}
\hline $\begin{array}{l}\text { Vehicle } \\
\text { Type }\end{array}$ & $\begin{array}{l}\text { Accel. }\left(\mathrm{ms}^{-2}\right) \\
\left(\mathrm{ms}^{-2}\right)\end{array}$ & $\begin{array}{l}\text { Decel. } \\
(\text { Sigma }\end{array}$ & $\begin{array}{l}\text { Max } \\
\text { Speed } \\
\left(\mathrm{ms}^{-1}\right)\end{array}$ & $\begin{array}{l}\text { Probability } \\
(\%)\end{array}$ \\
\hline \hline Fast & 4 & 6 & 0.2 & 36 & 10 \\
Normal & 2 & 4 & 0.3 & 28 & 80 \\
Slow & 2 & 4 & 0.5 & 20 & 10 \\
\hline
\end{tabular}

Note: Accel. and Decel. are stands for acceleration and deceleration, respectively.

A comparison of these results with the results for the urban environment shows that there is a negligible
Figure 7: PDR for the highway scenario.

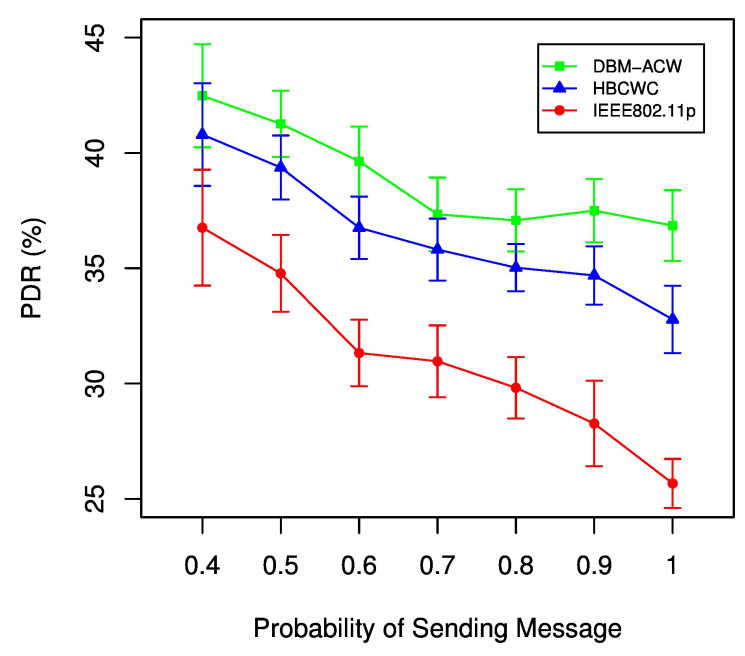

difference between highway and urban scenarios, as pointed out by Wellens et al. (2007). In the assumed highway scenario, the number of collisions is lower than in the urban scenario (due to the lower transmission rate), depicted in Figure 8. This figure shows that DBMACW improves the number of collisions, but the trend is not exactly the same as for the urban scenario, meaning that, for lower densities, DBM-ACW's performance is similar to HBCWC. Consequently, DBM-ACW cannot achieve a high improvement ratio compared with HBCWC in terms of PDR under lower densities, as depicted in Figure 7. However, for high densities, DBMACW achieves a higher improvement ratio compared to HBCWC when simultaneously considering both the PDR and the number of collisions. While DBM-ACW shows a lower improvement ratio when compared with the urban scenario, it still shows better results than HBCWC and IEEE 802.11p considering the PDR and the number of collisions.

In terms of end-to-end delay, DBM-ACW clearly outperforms IEEE 802.11p, as shown in Figure 9. Also, while IEEE 802.11p has an increasing trend, DBMACW shows a decreasing trend, meaning that our approach does not increase the delay when the number of connections increases, as desired.

Figure 9 shows that HBCWC achieves a slightly better delay when compared with DBM-ACW. However, DBM-ACW achieves a better trade-off between PDR and end-to-end delay, meaning that the total PDR improvement ratio is higher than the delay degradation ratio when compared with HBCWC.

Overall, we obtain a $20 \%$ improvement in terms of PDR, and a $22 \%$ improvement in terms of end-to-end delay compared with IEEE 802.11p, as well as a $7 \%$ improvement in PDR compared with HBCWC. 
Figure 8: Average number of collisions for the highway scenario.

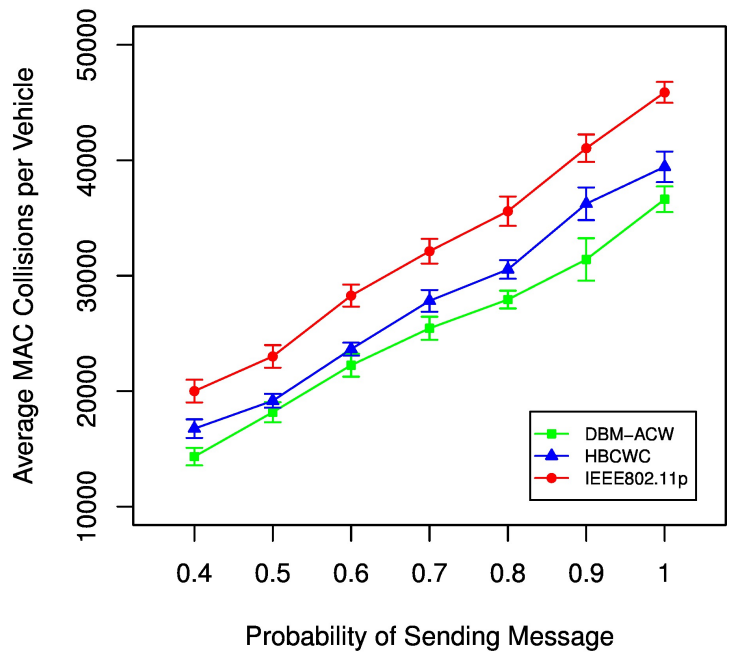

Figure 9: Average end-to-end delay for the highway scenario.

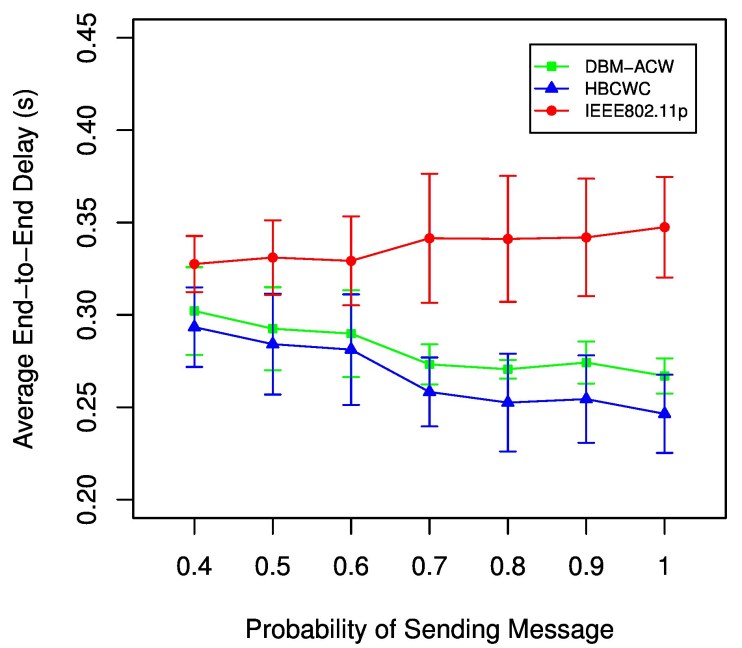

\section{Conclusion}

The CSMA-based medium access control protocol is the preferred method for the current generation of vehicular ad hoc networks. Although this scheme has its merits, its suffers from scalability problems in dynamic environments with high node density, like vehicular environments, that affects its performance. In this paper we demonstrate the impact of contention window adjustments on the overall performance of unicast communications in vehicular ad hoc networks.
The key contribution of this paper is to improve the scalability of IEEE 802.11 p by dynamically adapting the $\mathrm{CW}$ size in vehicular environments. In our DBM-ACW approach, the channel status is observed and used in order to dynamically tune the CW size. The critical part of DBM-ACW is how the CW size is adjusted based on the channel status array.

Extensive simulation results prove that our scheme shows better overall performance when compared with IEEE 802.11p and HBCWC, improving the PDR and the end-to-end delay for different vehicular environments, which was the main goal of this paper.

As a future work, we will study the control channel transmission challenges, and we will propose a new collision-free channel access scheme for broadcast communications in the control channel.

\section{References}

Aad, I., Qiang Ni, Barakat, C., Turletti, T. (2004). Enhancing IEEE 802.11 MAC in congested environments. in Proc. ASWN 2004, pp. 82-91, Aug. 2004.

Asadallahi, S., Refai, H.H. (2012). Modified R-ALOHA: Broadcast MAC protocol for Vehicular Ad hoc Networks. in Proc. IWCMC, pp. 734-738, Aug. 2012.

Baguena, M., Calafate, C.T., Cano, J., Manzoni, P. (2012). Towards realistic vehicular network simulation models. in Proc. Wireless Days, pp. 1-3, Nov. 2012.

Baguena, M., Tornell, S., Torres, A., Calafate, C.T., Cano, J.C., Manzoni, P. (2013). VACaMobil: VANET Car Mobility Manager for OMNeT++. in Proc. SCPA, June 2013.

Bai, F., Elbatt, T., Hollan, G., Krishnan, H., Sadekar, V. (2006). Towards characterizing and classifying communication-based automotive applications from a wireless networking perspective. in Proc. Globecom workshop, Dec. 2006.

Balador, A., Movaghar, A., Jabbehdari, S. (2010). History based contention window control in ieee 802.11 mac protocol in error prone channel. Computer Science, vol. 6, no. 2, pp. 205-209, 2010.

Behrisch, M., Bieker, L., Erdmann, J., Krajzewicz, D. (2011). SUMO - Simulation of Urban MObility: An Overview. in Proc. SIMUL, Oct. 2011.

Bharghavan, V., Demers, A., Shenker, S., Zhang, L. (1994). MACAW: A media access protocol for wireless LANs. in Proc. SIGCOMM, United Kingdom, pp. 21225, Aug. 1994.

Bianchi, G., Tinnirello, I. (2003). Kalman filter estimation of the number of competing terminals in an IEEE 802.11 network. in Proc. INFOCOM, vol. 2, pp. 844-852, Apr. 2003. 
Bohm, A., Lidstrom, K., Jonsson, M., Larsson, T. (2010). Evaluating CALM M5-based vehicle-to-vehicle communication in various road settings through field trials. in Proc. LCN, Oct. 2010.

Bononi, L., Conti, M., Gregori, E. (2004). Runtime optimization of IEEE 802.11 wireless LANs performance. IEEE Trans. on Parallel and Distributed Systems, vol. 15, no. 1, pp. 66-80, Jan. 2004.

Booysen, M.J., Zeadally, S., van Rooyen, G.-J. (2011). Survey of media access control protocols for vehicular ad hoc networks. IET Communications, vol. 5, no. 11, pp. 1619-1631, July 2011.

Cali, F., Conti, M., Gregori, E. (2000). Dynamic tuning of the IEEE 802.11 protocol to achieve a theoretical throughput limit. IEEE/ACM Trans. on Networking, vol. 8, no. 6, pp. 785-799, Dec. 2000.

Chrysostomou, C., Djouvas, C., Lambrinos, L. (2011). Applying adaptive QoS-aware medium access control in priority-based vehicular ad hoc networks. in Proc. ISCC, pp. 741-747, July 2011.

Clifford Neuman, B. (1994). Scale in Distributed Systems, Readings in Distributed Computing Systems. IEEE Computer Society Press, 1994.

Cottingham, D.N., Wassell, I.J., Harle, R.K. (2007). Performance of IEEE 802.11a in Vehicular Contexts. in Proc. VTC2007-Spring, pp. 854-858, Apr. 2007.

Grafling, S., Mahonen, P., Riihijarvi, J. (2010). Performance evaluation of IEEE 1609 WAVE and IEEE 802.11p for vehicular communications. in Proc. ICUFN, pp. 344-348, June 2010.

Hartenstein, H., Laberteaux, K.P. (2008). A tutorial survey on vehicular ad hoc networks. IEEE Communications Magazine, vol. 46, no. 6, pp. 164-171, June 2008.

Heusse, M., Rousseau, F., Guillier, R., Duda, A. (2005). Idle Sense: An Optimal Access Method for High Throughput and Fairness in Rate Diverse Wireless LANs. in Proc. SIGCOMM, Aug. 2005.

IEEE 802.11p Std-2012. IEEE Standard for Information technology Telecommunications and information exchange between systems Local and metropolitan area networks Specific requirements Part 11: Wireless LAN Medium Access Control (MAC) and Physical Layer (PHY) Specifications Amendment 6: Wireless Access in Vehicular Environments.

ANSI/IEEE Std 802.11-2003. IEEE Standard for Information Technology Telecommunications and Information Exchange Between Systems Local and Metropolitan Area Networks Specific Requirements Part 11: Wireless LAN Medium Access Control (MAC) and Physical Layer (PHY) Specifications.
IEEE Std 802.11e-2005. IEEE Standard for Information technology Local and metropolitan area networks Specific requirements Part 11: Wireless LAN Medium Access Control (MAC) and Physical Layer (PHY) Specifications - Amendment 8: Medium Access Control (MAC) Quality of Service Enhancements.

Eichler, S. (2007). Performance Evaluation of the IEEE 802.11p WAVE Communication Standard. in Proc. VTC-2007Fall. pp. 2199-2203, Oct. 2007.

Jansons, J., Barancevs, A. (2012). Using wireless networking for vehicular environment: IEEE 802.11a standard performance. in Proc. ICDIPC, pp. 5-9, July 2012.

Kenney, J. (2010). Standards and regulations. in Hartenstein, H., Laberteaux, K.P. (Eds.): VANET: vehicular applications and inter-networking technologies (Wiley, 2010). Ch. 10, pp. 365-428.

Kenney, J.B. (2011). Dedicated Short-Range Communications (DSRC) Standards in the United States. Proceedings of the IEEE, vol. 99, no. 7, pp. 1162-1182, July 2011.

Martinez, F., Fogue, M., Toh, C. K., Cano, J.-C., Calafate, C. T., Manzoni, P. (2013). Computer simulations of VANETs using realistic city topologies. Wireless Personal Communications, vol. 69, no. 2, pp. 639-663, Mar. 2013.

Omar, H., Zhuang, W., Li, L. (2012). VeMAC: A TDMA-based MAC Protocol for Reliable Broadcast in VANETs. IEEE Trans. on Mobile Computing, vol. 12, no. 9, pp. 1724-1736, Sept. 2013.

Qiang Ni, Aad, I., Barakat, C., Turletti, T. (2003). Modeling and analysis of slow CW decrease IEEE 802.11 WLAN. in Proc. PIMRC, vol.2, pp. 1717-1721, vol. 2, Sept. 2003.

Stanica, R., Chaput, E., Beylot, A.-L. (2011). Enhancements of IEEE 802.11p Protocol for Access Control on a VANET Control Channel. in Proc. ICC, pp. 1-5, June 2011.

Sommer, C.; Eckhoff, D.; German, R.; Dressler, F. (2011). A computationally inexpensive empirical model of IEEE $802.11 p$ radio shadowing in urban environments. in Proc. WONS, pp. 84-90, Jan. 2011.

Song, N.-O., Kwak, B.-J., Song, J., Miller, M.E. (2003). Enhancement of IEEE 802.11 distributed coordination function with exponential increase exponential decrease backoff algorithm. in Proc. VTC 2003-Spring, vol. 4, pp. 2775-2778, Apr. 2003.

Wang, S.-S., Chen, H.-C., Chang, J.-K. (2012). A distributed adaptive MAC protocol for efficient broadcasting in vehicular ad hoc networks. in Proc. WCNC, pp. 1555-1560, Apr. 2012. 
Wellens, M., Westphal, B., Mahonen, P. (2007). Performance Evaluation of IEEE 802.11-based WLANs in Vehicular Scenarios. in Proc. VTC2007Spring, pp. 1167-1171, Apr. 2007.

Wu, H., Cheng, S., Peng, Y., Long, K., Ma, J. (2002). IEEE 802.11 distributed coordination function (DCF): analysis and enhancement. in Proc. ICC, vol. 1, pp. 605-609, Apr. 2002.

Zeadally, S., Hunt, R., Chen, Y.S., Irwin, A., Hassan, A. (2012). Vehicular ad hoc networks (VANETS): status, results, and challenges. Telecommunication Systems, vol. 50, no. 4, pp. 217-241, Aug. 2012.

\section{Websites}

INETMANET webpage, http://inet.omnetpp.org (accessed on Nov. 2013).

OMNeT++ webpage, http://www.omnetpp.org (accessed on Nov. 2013).

OpenStreetMap website, http://www.openstreetmap.org (accessed on Nov. 2013). 